\title{
Teaching Functional Clinical Anatomy to Students in Medical and Health Sciences
}

\author{
Vian Mohialdin, Ari Shali and Hallie Groves
}

\begin{abstract}
In clinical practice, a thorough knowledge and understanding of functional clinical anatomy is fundamental in distinguishing between normal and pathology. For students in the first year of medicine and other health care professions, the integrated use of a range of learning resources, such as prosected human specimens, radiological images and the living human, can enhance learning and help develop an understanding of the clinical application of information. The synthesis of information from patient physical exam and radiological images are often essential components in developing a differential diagnosis, and in deciding on the best care and treatment for a patient.
\end{abstract}

Key words- anatomy, clinical skills, integration, first year medical students, education framework.

\section{INTRODUCTION}

It is a challenge to facilitate the development of the knowledge and understanding of functional clinical anatomy that is essential in clinical practice. Students in programs such as undergraduate and postgraduate medicine, physiotherapy and occupational therapy invest significant amounts of time learning anatomy by studying texts, dissected material and digital resources. However, early in their studies they often find it challenging to appreciate the clinical significance of the information and its application.

In the Anatomy Program at McMaster University, the use of an integrated range of resources including physical examination and radiological imaging (ultrasound, CT scans, x-rays and MRI), enhances learning and the appreciation of the clinical application of the information.

\section{PURPOSE}

The purpose of integrating the teaching of anatomy, other basic sciences such as physiology, applied clinical skills, diagnostic imaging and pathology is to facilitate and focus student learning, as well as enhance their understanding of the clinical application of knowledge.
The authors suggest anatomy is best learned through a 3 tiered system. (A know-can-do pyramid was first introduced by George Miller*. The idea is that medical competency is the result of a three tiered pyramid, where knowledge is the base, clinical skills come next and finally students are prepared to apply their knowledge and skills in clinical practice.)

\section{MATERIALS}

A series of 8 figures provide comparative detail from each of 4 modalities (cadaver, standardized patient, pathological specimen and radiological image) for the torso and forearm. The intent was to teach students how to integrate shape, location, size, orientation and dimensionality. The task of discriminating between normal and abnormal features is less daunting when students have a solid understanding of normal structure.

\section{METHODS}

The research we report in this manuscript is a 'preliminary qualitative study' and provides the template for a theoretical model for teaching clinical anatomy.

The Medical Program at McMaster University is a 3 year program divided into 5 foundations, each with a specific focus. Throughout the program the learning of functional clinical anatomy and its application is organized into three tiered hierarchy and compliment with the 'health care problems', The clinical skills practice sessions hand on a warm body (standardized patients) are coordinated with an anatomy lab using human cadavers material. Finally, actual patients are examined.

For example, in foundation which focuses on the cardiovascular and respiratory systems, medical students will have three scheduled Anatomy/Clinical Skills sessions. The Anatomy lab session will focus on the detailed anatomy of the thorax including the chest wall, diaphragm, contents of the thoracic cavity, organs and their location and relationships, as well as important landmarks and surface anatomy. The students will then have a clinical skills session with 
clinical teaching faculty and a standardized patient. Here the students focus on the integration of their anatomical knowledge and clinical examination skills, as they learn where to place the stethoscope on a patient's chest wall when they auscultate the heart sounds (Figures 1A, and 1B) or where to feel for the radial pulse ( Figure 3A and 3B).

When students are studying the gastrointestinal system, they first locate the liver in a human cadaver, find its relation to abdominal viscera, lobes, size and surface marking (Figures 2A and 2B), and then learn how to palpate a tender liver in a patient with liver problem.

In addition, relevant radiological images and pathology specimens are integrated into the anatomy/clinical skills teaching sessions. The examples shown include a chest X-ray with a consolidation (figure 4), and a thyroid specimen with cancer and one without cancer for comparison (figure 5).

Student comments were collected at the end of these sessions in order to determine the effectiveness of these sessions on their learning.

\section{RESULTS}

Student comments strongly supported the integrated teaching model comprised of both basic anatomy and clinical skills, and felt that they laid the foundation for optimal learning. Examples of the students' comments include:

"I believe that the teaching we have received by the faculty at the Anatomy/Clinical Skills lab has been a crucial part of my curriculum as a medical student. The teaching style is, I believe, unique to McMaster and has always been very clinically relevant. Prosected specimens and cadavers, as well as gross pathological specimens are incorporated into each anatomy session, which has been wonderful since it has helped to consolidate what we have learned elsewhere on certain disease states".

and,

"The discussions usually delve into the clinical presentation of certain diseases and are linked with the gross pathology specimens that are presented to the group; this later approach has enhanced my understanding of the pathophysiology behind several disease states. In addition, radiology is usually incorporated into most sessions, including plain films, ultrasound, CT, MR imaging and more specific contrast or functional imaging studies. I found this aspect to be very helpful in learning how to correlate radiological findings with anatomy and anatomical /functional abnormalities. Furthermore, the fundamentals that $\mathrm{I}$ was taught in interpreting radiographic images have proven to be useful in the hospital wards during my clerkship rotations. In summary, I am convinced that the Anatomy Program at McMaster has helped to reinforce not only my anatomy knowledge, but also a basic understanding of disease states and in the radiological presentation of the same".

\section{NOTES ON CONTRIBUTORS}

\section{Vian Mohialdin, MBChB}

Assistant professor

Department of Pathology and Molecular Medicine

Division of Anatomy, McMaster University

Hamilton, Ontario, Canada.

Dr. Mohialdin teaches Anatomy, Radiology and Clinical Skills to $1^{\text {st }}, 2^{\text {nd }}$ and $3^{\text {rd }}$ year Medical students, and for Residency Program.

\section{Ari Shali, MBChB}

Assistant professor

Department of Pathology and Molecular Medicine

Division of Anatomy, McMaster University

Hamilton, Ontario, Canada

Dr. Shali teaches Anatomy and Clinical Skills to $1^{\text {st }}$, $2^{\text {nd }}$, and $3^{\text {rd }}$ year Medical students, and for Residency Program.

Hallie Groves, $\mathrm{PhD}$.

Professor Emeritus

Department of Pathology and Molecular Medicine

Hamilton, Ontario, Canada

\section{Mary Lou Schmuck}

ACKNOWLEDGEMENTS

Sharon Ralph 

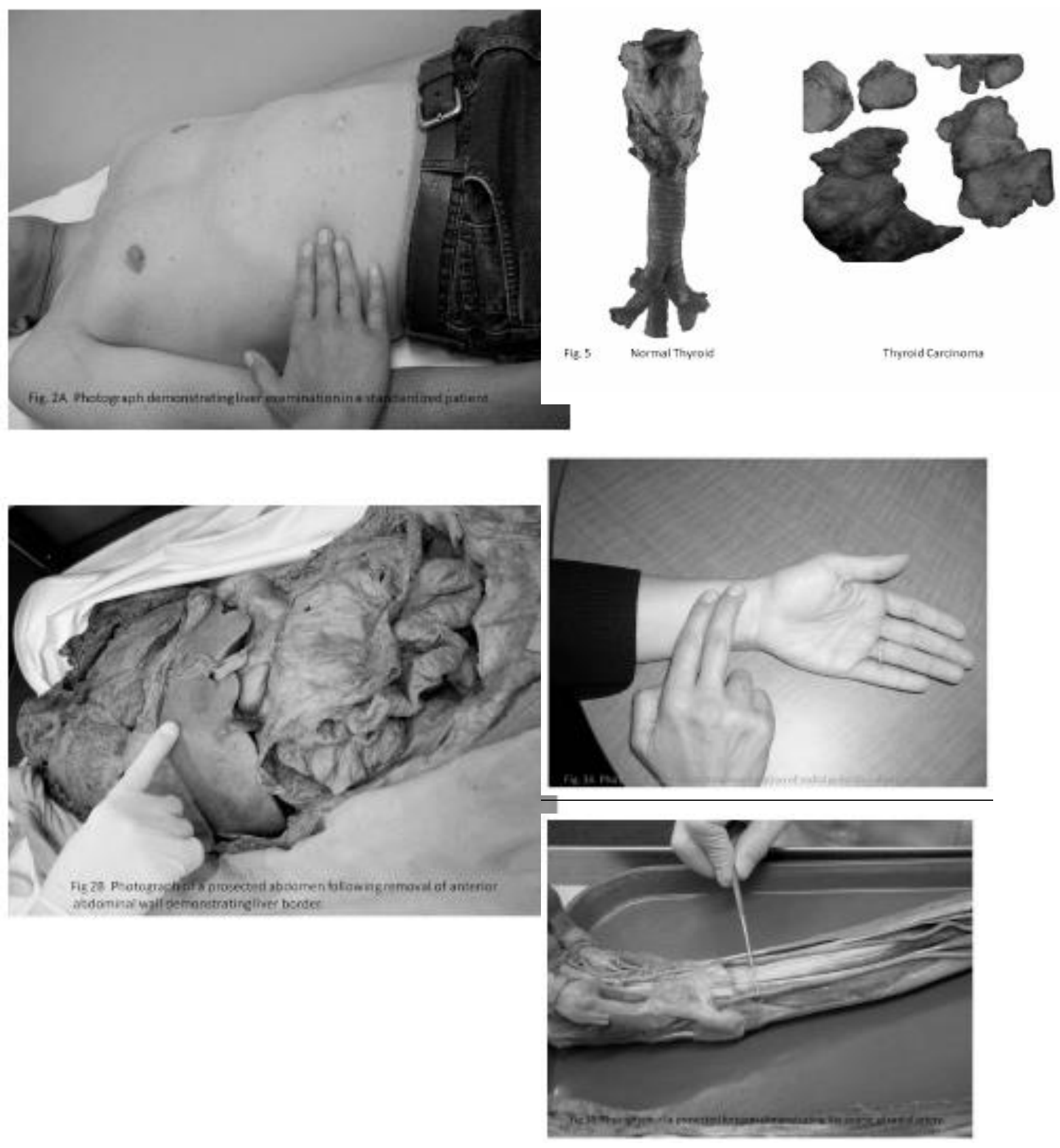

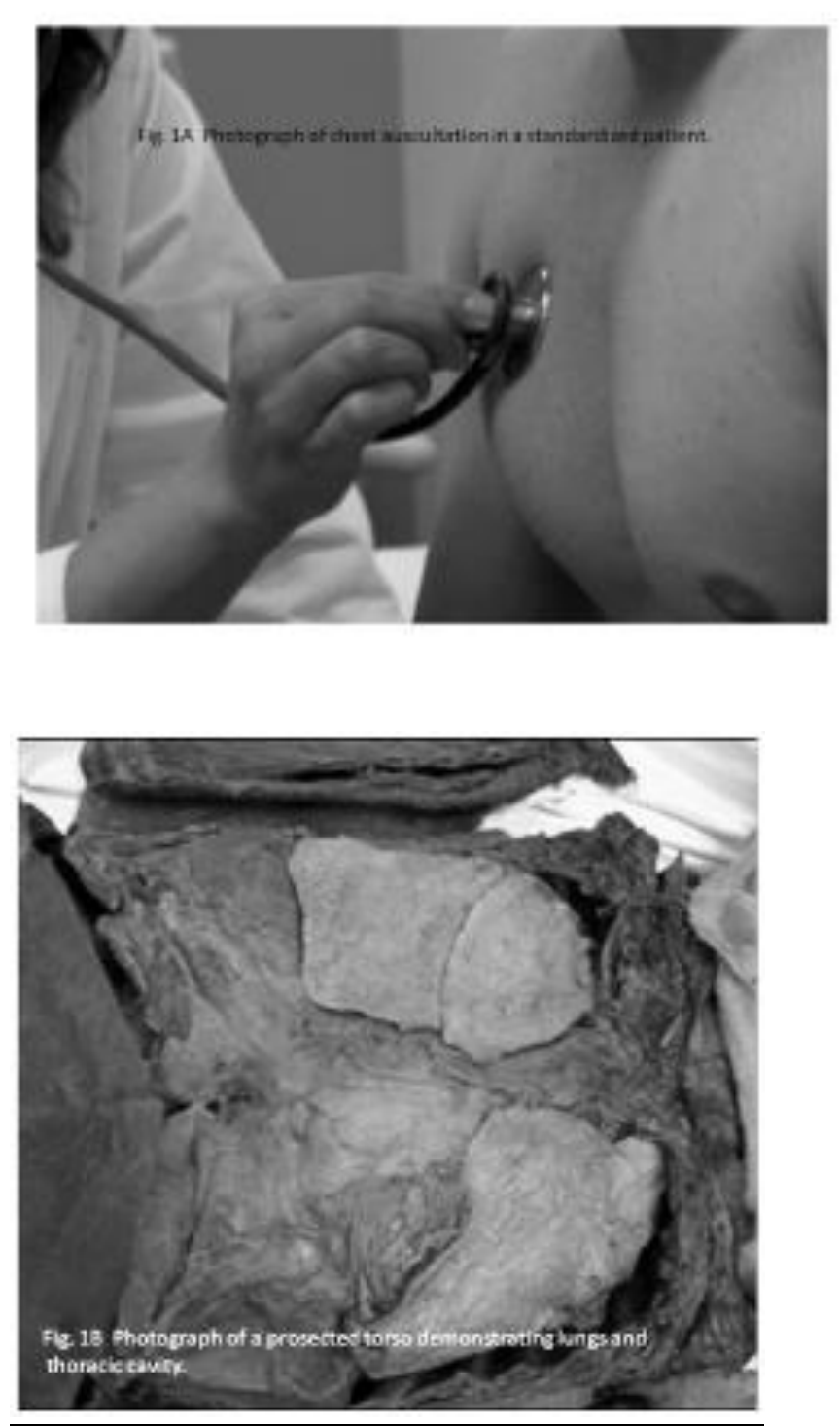

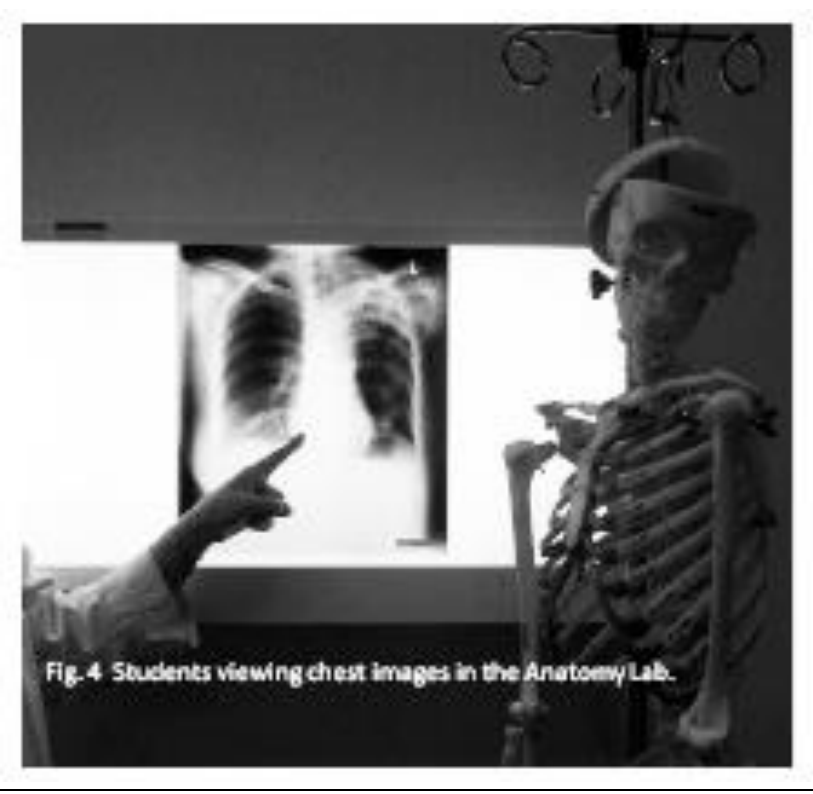

\section{REFERENCES}

Cowan, M., Arain, N.N., Abu Assale, T.S., Assi, A.H., Albar, R.A., Ganguuly, P.K. (2010). Student-centered integrated anatomy resources sessions at Alfaisal University. Anatomical Sciences Education, 3, 272-275.

Drake, R.L. (2007). A unique, innovative, and clinically oriented approach to anatomy education. Academic Medicine, 82(5), 475478.

El-Moamly, A.A. (2008). Problem-based learning benefits for basic sciences education. Anatomical Sciences Education. 1, 189-190.

*Miller, G.E. (1990). The assessment of clinical skills/competence performance. Academic Medicine S63-67.

Netterstrom, I., Kayser, L. (2008). Learning to be a doctor while learning anatomy! Anatomical Sciences Education. 1, 154-158. Doi:10.1002/ase.31.

Neville, A.J., Norman, G. (2007). PBL in the undergraduate MD program at McMaster University: three iterations in three decades. Academic Medicine, 82(4), 370-374.

Wittert, G.A., Nelson, A.J. (2009). Medical education: revolution, devolution and evolution in curriculum philosophy and design. Med J Aust. 6, 191(1), 35-7.

Wood, A., Struthers, K., Whiten, S., Jackson, D., Simon Herrington C. (2010). Introducing gross pathology to undergraduate medical students in the dissecting room. Anatomical Sciences Education. $3(2), 97-100$.

Zumwalt, A.C., Marks, L., Halperin, E.C. (2007). Integrating gross anatomy into a clinical oncology curriculum: the oncoanatomy course at Duke University School of Medicine. Academic Medicine. 82(5), 469-474. 


\section{AUTHORS' PROFILE}

Dr. Vian Mohialdin.

Graduated from University of Baghdad, $\mathrm{MBChB}$ degree received in 1986.

M.Sc. degree in Radiology received in 1990.

Joined McMaster University in 1992 as a lecture in the department of Biomedical Sciences.

Currently is an Assistant Professor at the department of Pathology and Molecular Medicine, McMaster University.

Heavily involved in teaching Medical Program, residency program, Physiotherapy program, and Physician Assistant Program.

Member of the Association of Faculties of Medicine of Canada, ACMC.

Member of Canadian National Clinical Skills Interest group, AFMC.

Member of Canadian Association of Anatomy.

Member of American Association of Anatomy.

Dr. Ari Shali is an Assistant Professor in the Department of Pathology and Molecular Medicine. He Joined McMaster University in 1993 as a lecturer in the Department of Biomedical Sciences. Dr. Shali is heavily involved in teaching in the following programs: MD, Physician Assistant and Residency Programs. He was the Chair of Clinical Skills for the MD Program from 2001-2014 and he is also a member of many committees in the MD program. Dr. Shali is also a member of the following: Association of Faculties of Medicine of Canada (AFMC), a member of AFMC National Clinical Skills Interest Group and Canadian Association for Anatomy, Neuroanatomy and Cell Biology (CAANCB).

Hallie Groves, PhD is a Professor Emeritus in the Department of Pathology and Molecular Medicine Hamilton, Ontario, Canada. 